\title{
Patient satisfaction analysis after interaction with emergency department clinical staff through interpreters
}

\author{
Muhammad N Qureshi' ${ }^{1}$,Johara Alameel ${ }^{2}$, Bashayer Almousa ${ }^{2}$, Abdul Moneim Eldali ${ }^{3}$ and Taimur S Butt MD ${ }^{1}$ \\ ${ }^{1}$ Department of Emergency Medicine, King Faisal Specialist Hospital and Research Centre, KSA \\ ${ }^{2}$ College of Medicine, Al Faisal University, KSA \\ ${ }^{3}$ Department of Biostatistics, Epidemiology and Science Computing Department, Research Centre, King Faisal Specialist Hospital and Research Centre, KSA
}

\begin{abstract}
Background: Worldwide, medical professionals use onsite or remote interpreter services via phone to communicate with their patients. In the Middle-East, majority of the non-Arabic Speaking Emergency Department (ED) staff use in-house interpreters.

Objective: Measure patient satisfaction in adults (>14 years) after clinical interaction with the interpreter during an Emergency encounter.

Design: We conducted a cross-sectional study where a random sample of adult ED patients were recruited to participate in a patient satisfaction questionnaire at the end of their clinical encounter. Hospital approved questionnaire (Project \#2171091) was completed by the patients, their attendants or investigators on behalf of the patients.
\end{abstract}

Setting: Tertiary Care ED in the Middle East.

Patients: All ED patients aged 14 and above were eligible.

Main outcome measures: ED patient satisfaction at the end of a clinical encounter with the involvement of an interpreter.

Sample size: A comparative analysis was conducted between 184 patient encounters, who consented to enroll in the study. Ten patients did not consent to participate.

Results: The questionnaire was completed by the patients or their family members in 160 (87\%) subjects or unrelated volunteers assisted 24 (13\%) subjects to complete the data forms. A total of 173 (94\%) patients were Saudi Arabian nationals. Out of the 77(42\%) patients who used interpreters, 28 (36.3\%) used more than one interpreter during a clinical encounter. Out of the 77 (42\%) patients who used an interpreter during the clinical encounter, 62 (80.5\%) patients believed that their doctors and/or nurses understood their concern $(P>.05)$.

Limitations: Overcrowding and delay in patient care could have influenced patient satisfaction.

Conclusions: Use of one or more interpreters by Limited Arabic Speaking (LAP) staff during a single clinical encounter did not adversely affect patient satisfaction in our ED.

\section{Introduction}

Worldwide, medical professionals may use in house interpreter services or via phone to communicate with their patients, who have limited English proficiency (LEP). Approximately $50 \%$ of the clinicians and $67.7 \%$ nurse workforce working in Kingdom of Saudi Arabia (KSA) do not have their first language as Arabic [1].

ED medical staff at our tertiary care ED, like most other hospitals in the KSA are diverse in their linguistic capabilities but may have limited Arabic Proficiency (LAP) [1]. Most of the patients presenting to the ED are Native Arabic language speaking with LEP. In the KSA, majority of the LAP Emergency Department (ED) staff use in-house interpreters to communicate with the patients and families during clinical encounters [2]. A clinical encounter is a direct medical practitioner to patient interaction, with a view to provide optimal clinical care, which involves consideration of the patient's culture [3].

Cultural competency has been defined as an understanding of all manifestations of social diversity, an important element of which is satisfactory communication with patients and families regardless of the linguistic proficiency [4]. Cultures have been broadly classified as High and Low Context Cultures with different styles of communication. The KSA culture falls under High Context by this definition; the patients convey the main part of the message through non-verbal communication and simple verbal messages have deeper meanings $[5,6]$. In contrast, the Low Context (Western) Cultures tend to adopt a direct way of communication with explicitness in conversation. Therefore, a native language speaking interpreter is vital for optimal patient to provider communication.

${ }^{*}$ Correspondence to: Muhammad Qureshi, Consultant, Department of Emergency Medicine, King Faisal Specialist Hospital and Research Centre, PO Box 3354, Riyadh 11211, Kingdom of Saudi Arabia, Tel: 966-11-4424425; E-mail: qmuhammad@kfshrc.edu.sa

Key words: interpreter, communication, Emergency Department (ED)

Received: January 30, 2019; Accepted: February 11, 2019; Published: February 13, 2019 
Many studies in the western countries have shown that language non-proficiency may affect the quality of medical care delivered to the patients and they are more likely to use ED for non-emergency care services [6]. It has also been shown that language hurdles may adversely affect patient ability to understand their medical conditions and therefore directly influence patient compliance with treatment and follow-up appointments [7].

In the English-speaking countries, the LEP group expressed decreased satisfaction scores to communication, explanation offered about their medications, reassurance and support [8]. They were also less satisfied with courtesy, completeness of care, waiting times, discharge instructions and what was done for them as compared to English speakers [9].

\section{Methodology}

The study was approved by the Research Advisory Council (RAC) of our hospital in, Riyadh, KSA and it abides by the laws of the Kingdom of Saudi Arabia. It was conducted from June to October 2017.

A descriptive study method was used for the research design in which questionnaire were filled by the patients, their family members or unrelated volunteers proficient in English and Arabic languages. A comparison of ED patient encounters between Arabic and non-Arabic speaking nurses and clinicians was studied.

A validated Arabic patient satisfaction questionnaire approved by the Research Advisory Council (RAC) was used for data collection. The calculated Cronbachs alpha for the satisfaction questions is 0.83 . Various patient satisfaction domains were targeted. Patients became eligible for the study after signing an informed consent at the end of the clinical encounter. For study purposes, an ED clinical encounter ended, when patient was either referred to a specialty, admitted or discharged.

Two investigators who are also the coauthors, recruited the patients. Simple random sampling was used for enrolment. Study recruitment was not restricted to any specific time or day of the week. Medical record numbers were used as patient identifiers.

Patients were informed that study participation is voluntary, will not affect their current or future clinical encounters and is in accordance with the patients' bill of rights. The collected data will be kept confidential and used for research purposes only.

The statistical analysis of this study was done by using the software package SAS version 9.4 (SAS Institute Inc., Cary, NC, USA). Descriptive statistics for the continuous variables are reported as mean \pm standard deviation and categorical variables are summarized as frequencies and percentages.

The categorical variables are compared by Chi-square test and the continuous variables are compared by Student's independent $t$-test.

The level of statistical significance is set at $p<0.05$.

\section{Results}

The predominant study population was Saudi citizens who have Arabic as their native language. The study sample did not have any significant gender, age or level of education difference between the two groups (interpreter vs. No interpreter used). Similarly, the recruitment of subjects, CTAS distribution of patients, patient level of education and other variables were not significantly different. However, more than one interpreter was used by the medical staff during the same clinical encounter $(P<.0001)$. (Table 1$)$.
Table 1. Demographics

\begin{tabular}{|c|c|c|c|}
\hline & Interpreter Used & No Interpreter Used & P-value \\
\hline Item & Count (\%) & Count (\%) & \\
\hline \multicolumn{4}{|l|}{ Gender: } \\
\hline Female & $46(42.99)$ & $61(57.01)$ & \\
\hline Male & $31(40.26)$ & $46(59.74)$ & 0.7111 \\
\hline \multicolumn{4}{|l|}{ Nationality: } \\
\hline Saudi & $73(42.20)$ & $100(57.80)$ & \\
\hline Non-Saudi & $4(36.36)$ & $7(63.64)$ & 0.7038 \\
\hline \multicolumn{4}{|l|}{ Residence: } \\
\hline Riyadh & $46(37.40)$ & $77(62.60)$ & \\
\hline Outside Riyadh & $31(50.82)$ & $30(49.18)$ & 0.0823 \\
\hline \multicolumn{4}{|l|}{ Education: } \\
\hline None & $16(8.38)$ & $24(61.54)$ & \\
\hline Elementary & $15(40.0)$ & $18(60.0)$ & \\
\hline High School & $13(44.83)$ & $16(55.17)$ & \\
\hline University & $26(42.37)$ & $34(57.63)$ & \\
\hline Postgraduate & $3(50.0)$ & $3(50.0)$ & \\
\hline Other & $7(43.75)$ & $9(56.25)$ & 0.9912 \\
\hline \multicolumn{4}{|l|}{ Age: } \\
\hline$<14$ Years & $6(20.0)$ & $24(80.0)$ & \\
\hline 14-20 Years & $5(35.71)$ & $9(64.29)$ & \\
\hline 21-40 Years & $28(45.9)$ & $33(54.1)$ & \\
\hline 41-65 Years & $28(47.46)$ & $31(52.54)$ & \\
\hline 66-80 Years & $8(47.06)$ & $9(52.94)$ & \\
\hline$>80$ Years & $2(66.67)$ & $1(33.33)$ & 0.1443 \\
\hline \multicolumn{4}{|l|}{ Shift Time: } \\
\hline Morning & $32(53.33)$ & $28(46.67)$ & \\
\hline Evening & $34(35.05)$ & $63(34.81)$ & \\
\hline Night & $10(41.67)$ & $17(58.33)$ & 0.0786 \\
\hline \multicolumn{4}{|l|}{ CATS Category: } \\
\hline 1 & $4(100)$ & 0 & \\
\hline 2 & $10(29.41)$ & $24(70.59)$ & \\
\hline 3 & $39(38.61)$ & $62(61.39)$ & \\
\hline 4 & $24(54.55)$ & $20(45.45)$ & \\
\hline 5 & 0 & $1(100)$ & 0.0606 \\
\hline \multicolumn{4}{|l|}{$\begin{array}{l}\text { More than one } \\
\text { interpreter used: }\end{array}$} \\
\hline Yes & $27(96.43)$ & $1(3.57)$ & \\
\hline No & $45(30.82)$ & $101(69.18)$ & $<.0001$ \\
\hline
\end{tabular}

The understanding of the patients' diagnosis \& concerns by the ER staff and explanation offered to patients by the ER staff was adequately understood, without any significant difference between the groups. There was also no significant difference in the overall satisfaction between the two groups $(P<.70)$. (Table 2$)$.

The survey received a total of 184 responses from patients; out of 184 patients in the study, 108 (58.6\%) were female and 78 (42.3\%) were male.

14 (7.6\%) were aged between 14-20 yrs., 61 (33.1\%) were aged between $21-40$ yrs., 59 (32\%) were aged between $41-65$ yrs., 17 (9.2\%) were between the age of $66-80 \mathrm{yrs}$., 30 (16.3\%) were less than $14 \mathrm{yrs}$. old and $3(1.6 \%)$ were more than 80 years of age.

174 (94.5\%) were Saudi Arabian citizens and 122 (65.3\%) were from Riyadh region. 78 (42.3\%) patients competed the questionnaire themselves, patients' families completed 81 (44\%), and 24 (13\%) questionnaires were completed by either of the two study investigators, on behalf of the patients.

The study population level of education; $6(3.2 \%)$ had a postgraduate degree, 60 (32.6\%) had attended university, 29 (15.7\%) had 
Table 2. Statistical comparison of responses from the patients

\begin{tabular}{|c|c|c|c|}
\hline & Interpreter Used & No Interpreter Used & P-value \\
\hline Item & Mean \pm STD & Mean \pm STD & \\
\hline Your nurse understood your concern & $3.7(0.7)$ & $3.8(0.4)$ & 0.162 \\
\hline Your ER doctor understood your concern & $3.7(0.8)$ & $3.9(0.4)$ & 0.031 \\
\hline You received adequate explanation about your concern & $3.6(0.8)$ & $3.8(0.6)$ & 0.0731 \\
\hline You understood the diagnosis for presenting symptoms & $3.6(0.7)$ & $3.7(0.5)$ & 0.2117 \\
\hline You were able to ask the nurse or the doctor all the questions about your presenting condition & $3.7(0.7)$ & $3.8(0.5)$ & 0.4022 \\
\hline You understood the treatment plan explained by ER staff & $3.6(0.7)$ & $3.7(0.7)$ & 0.7382 \\
\hline Overall satisfaction with ER staff communication in today's visit & $8.3(2.5)$ & $8.4(2.2)$ & 0.7093 \\
\hline
\end{tabular}

a high school certificate, $16(8.6 \%)$ had other education level such as a diploma, middle school or special education, 33 (17.9\%) attended only elementary school, and 40 (21.7\%) had no formal schooling.

28 (36\%) patients used more than one interpreter during the same clinical encounter. Most of the subjects 72 (93.5\%) agreed that their interpreter was courteous and respectful. One respondent noted that interpreters were inconsistently courteous and respectful.

\section{Ability of ER Nurses to understand patients' concerns?}

The majority of patients $150(81.5 \%)$ agreed that their nurse was able to understand their concern, $21(11.41 \%)$ were unsure, 6 $(3.26 \%)$ disagreed with the statement while 2 (1.08\%) strongly disagreed (Figure 1).

\section{Ability of the ER physicians to understand the patient concerns?}

Regarding the ER physician understanding of the patients' concerns; $156(84.78 \%)$ agreed that their ER physician was able to understand their concern, $19(10.32 \%)$ were unsure, $5(2.71 \%)$ disagreed and 3 (1.63\%) strongly disagreed (Figure 2).

Did patients receive adequate explanation about their concerns?

$143(77.72 \%)$ agreed that their concerns were adequately addressed and explained, 25 (13.58\%) were unsure, while 9 (4.89\%) disagreed with the above statement and 5 (2.71\%) strongly disagreed (Figure 3).

\section{Patient understanding of the diagnosis?}

$135(73.36 \%)$ agreed that they understood the diagnosis of the presenting symptoms, $36(19.56 \%)$ were unsure of whether or not they understood the diagnosis, 8 (4.34\%) disagreed and 2 (1.08\%) strongly disagreed (Figure 4).

\section{Ability to ask the nurses or doctors questions regarding their condition}

The majority of patients $156(84.78 \%)$ agreed that they were able to ask their care-provider questions regarding their problem, $14(7.6 \%)$ were unsure, on the other hand, $11(5.97 \%)$ of the subjects disagreed with the statement believing they were unable to do so, while 2 (1.08\%) strongly disagreed (Figure 5).

\section{Understanding of the treatment plan explained by the ER staff}

Most of the patients 139 (73.91\%) agreed that they understood it, there were $29(15.76 \%)$ who were unsure, $10(5.43 \%)$ who disagreed and $4(2.17 \%)$ who strongly disagreed with plan explained by the examining physician. (Figure 6)

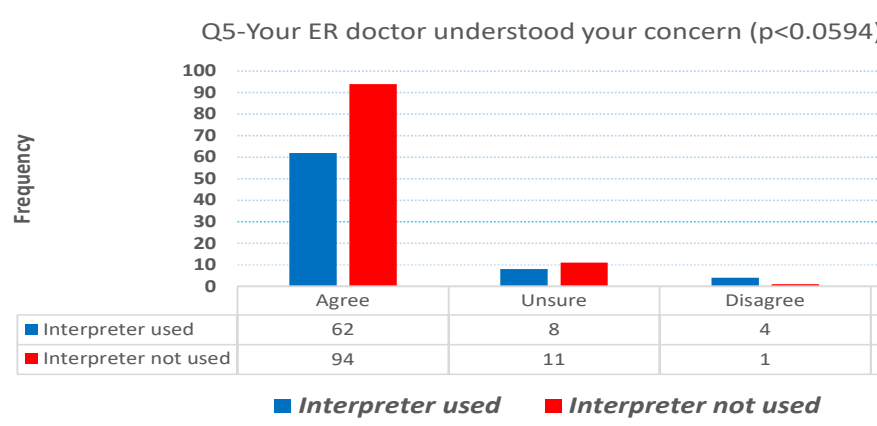

Figure 1. Ability of ER Nurses to understand patients' concerns.

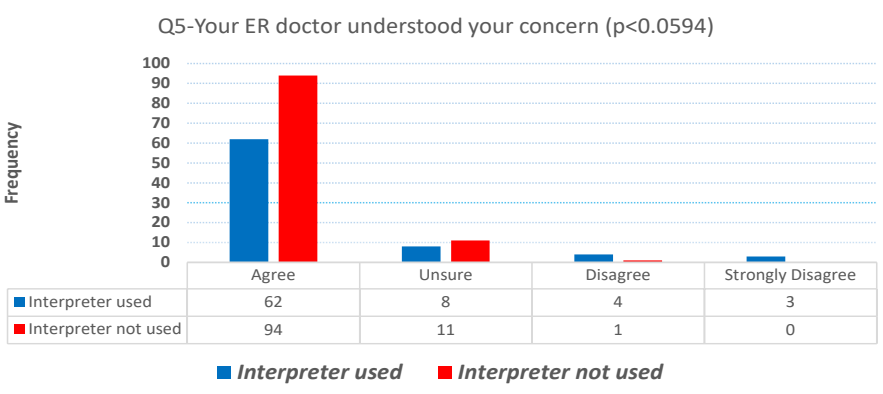

Figure 2. Ability of the ER physicians to understand the patient concerns.

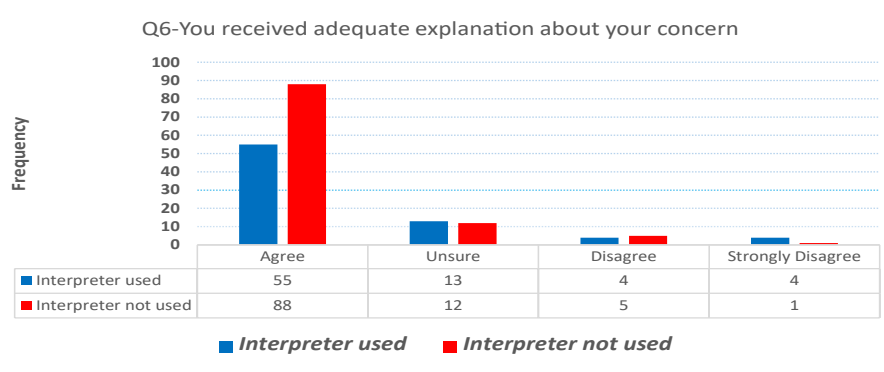

Figure 3. Did patients receive adequate explanation about their concerns?

Q7-You Understood the diagnosis for presenting symptoms $(p<0.3741)$

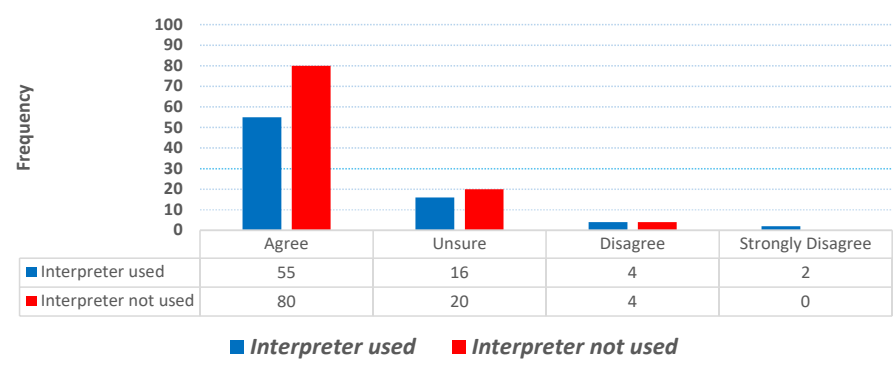

Figure 4. Patient understanding of the diagnosis? 
Q8-You were able to ask the nurse or the doctor all the questions about your presenting condition $(p<0.3769)$

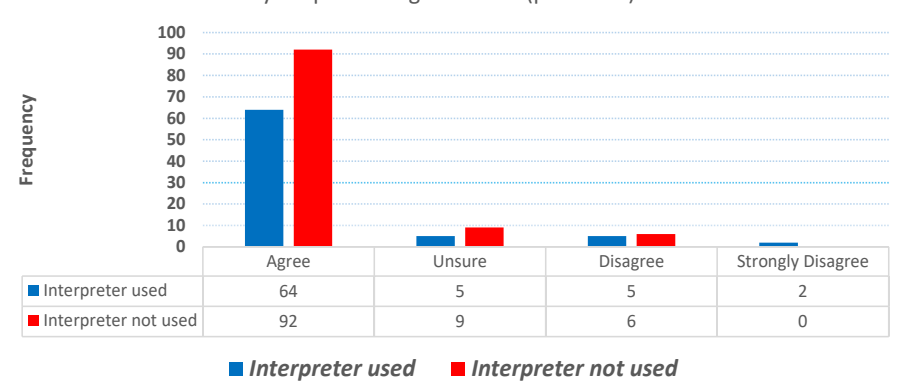

Figure 5. Ability to ask the nurses or doctors questions regarding their condition.

Q9-You understood treatment plan explained by the ER staff $(p<0.5749)$

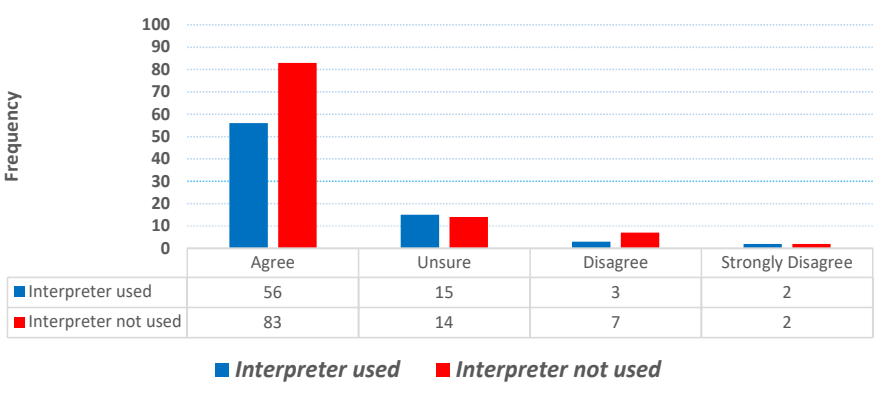

Figure 6. Understanding of the treatment plan explained by the ER staff.

\section{Overall satisfaction with ER staffs' communication}

On a score of 0-10 ( 0 being the least level of satisfaction and 10 equaling highest level of satisfaction), 94 (51\%) of the subjects scored their visit as $10 / 10$, twenty-four (13\%) scored it as $9 / 10$, twenty-two $(11.9 \%)$ scored it $8 / 10$, thirteen $(7 \%)$ gave it a $7 / 10$, ten $(5.4 \%)$ scored $6 / 10$, eleven $(5.9 \%)$ scored $5 / 10$. The 10 subjects who did not consent to the study, we assume their satisfaction score as zero.

\section{Discussion}

This study suggests that appropriate use of interpreters by LAP staff can attain similar patient satisfaction as with native language speaking staff. In our study, $94.56 \%$ of our subjects (174 respondents) scored between 5 to 10 on the overall ED staff communication satisfaction scale (Range 0-10; zero being least satisfied). The study was conducted in adult ED patients (age 14 and above). Most patients in our survey $(58.15 \% \mathrm{~N}=107)$ did not need an interpreter for their clinical encounter which could be due to the availability of Arabic speaking staff or family members acting as interpreters.

It is important for the interpreters to understand the patients' symptomatology and interpret it in a succinct way, as exact word to word translation of the patient's clinical symptoms may not be possible $[8,9]$.

Professional medical interpretation with trained interpreters is a specialized skill which is proven to be effective and has been established to be better than all other modalities experienced, e.g. Telephone based interpretations [10]. Although, majority of our senior physician and nursing staff are LAP but most of our patients did not require the use of an interpreter because of native arabic speeking junior staff who often where involved in the the clinical encounters and acted as interpreters. Although hospital professional inerpreters are provided with an accredited interpreter course but such linguistic training is not part of medical education for physician in the KSA. In our study, a relatively higher proportion of patients (12 with interpreter use vs 4 ), who utilized interpreter disagreed that they understood the treatment plan explained by the ED physician or nurse but this difference was not statistically significant $(P>.05)$. Our hospital interpreters do complete a medical terminology course before qualifying for the job. However, standardization of training that includes exposure to various English dialects and ED specific conversational scenarios should enable them to communicate better and enhance better patient understanding. Medical staff must also learn a standardized way of communication through the interpreter. A team working approach involving role playing and simulation may be deployed for effective learning as untrained interpreters can decrease the technical quality of interpretation, leading to more errors and omissions [11].

Patients' decisions made by the clinicians were delayed, observant and costlier, when LEP were managed without the involvement of trained interpreters [12]. In addition to the online resources, many hospitals in the region offer Medical Related Arabic Language Course which can improve communication skills for the LAP staff. However, this course is not a mandatory requirement. In a busy ED environment, lack of immediate availability of the interpreter can lead the clinician with basic knowledge of spoken Arabic to explain the treatment plan to the patient using their own limited grasp of native language (and sometimes with a combination of sign language), which can seriously impact patient's outcome [13]. Our results indicate that our patients were able to understand the diagnosis and the treatment plan provided by the ED physician and nursing staff through the interpreter.

It was interesting to note that the level of education in our study particepants who used or did not use an interpreter was not significantly different. It would appear that patients with limited schooling would normally use an interpreter. However, our results indicate that many patients with university and postgradate education required or opted to utilize interpretation services when dealing with ED staff with LAP. This might suggest that level of schooling may not correspond to the level of English language proficiency or Arabic speaking patients may feel more comfortable communicating in their native language. This observation may require further research.

The ED uses Canadian Triage Acuity Scale (CTAS) to categorize patients into five levels based on the severity of illness with level 1 as the sickest and level 5 as non-urgent. The largest group of patients fall under category 3 and 4 which is around $70 \%$ of our daily patient volume [14]. Our study cohort is therefore representative of our department population.

The study questions were derived from our hospital's validated patient satisfaction questionnaire. The study compared patient satisfaction across various domains during a single clinical encounter. It measured patient's satisfaction according to the standard Likert scale (Satisfaction score ranging from 1-10 or 'Not Satisfied' to 'Very Satisfied').

\section{Patient Satisfactory Survey form here}

\section{Limitations}

This survey was not designed to measure overall ED patient satisfaction from the clinical encounter. Recruitment of CTAS category 1 and 5 patients was limited due to lower volume or critical patient condition. Family members completing the survey could have influenced the satisfaction scores. The overcrowding and long waiting times in the ED may have also confounded patient satisfaction. The understanding of discharge instructions was not measured specifically. Patient waiting time related to the interpreter use was not measured. 


\section{Conclusion}

Use of one or more interpreters by LAP staff during a single clinical encounter in primarily Arabic speaking Middle Eastern population did not adversely affect patient satisfaction in an ED setting. In the study population, the level of schooling did not decrease interpreter utilization as many patients with university and postgraduate education required or opted to use interpretation services, when dealing with ED staff with LAP.

\section{Conflict of Interest}

None.

\section{References}

1. Ministry of Heath $1431 \mathrm{H}$, Health indicators, www.moh.gov.sa

2. Halligan P (2006) Caring for patients of Islamic denomination: critical care nurses' experiences in Saudi Arabia. Journal of clinical nursing 15: 1565-1573.

3. Tervalon M, Murray-Garcia J (1998) Cultural humility versus cultural competence: A critical distinction in defining physician training outcomes in multicultural education. Journal of health care for the poor and underserved 9: 117-125.

4. Cross TL (1989) Towards a Culturally Competent System of Care: A Monograph on Effective Services for Minority Children Who Are Severely Emotionally Disturbed. National Criminal Justice Reference Service.
5. Flores G, Laws BM, Mayo SJ, Zuckerman B, Abreu M, et al. (2003) Errors in medical interpretation and their potential clinical consequences in pediatric encounters. Pediatrics 111: 6-14.

6. Hampers LC, Cha S, Gutglass DJ, Binns HJ, Krug SE (1999) Language barriers and resource utilization in a pediatric emergency department. Pediatrics 103: 1253-1256.

7. González RD, Vásquez VF, Mikkelson H (1991) Fundamentals of court interpretation Durham, North Carolina: Carolina Academic Press.

8. Hewitt WE, Lee RJ (1996) Behind the language barrier, or You say you were eating an orange. State Court Journal 20: 23-31.

9. Gray B, Hilder J, Stubbe M (2012) How to use interpreters in general practice: the development of a New Zealand toolkit. J Prim Health Care 4: 52-61, A1-8. [Crossref]

10. Laws MB, Heckscher R, Mayo SJ, Li W, Wilson IB (2004) A new method for evaluating the quality of medical interpretation. Med Care 42: 71-80. [Crossref]

11. Hampers LC, McNulty JE (2002) Professional interpreters and bilingual physicians in a pediatric emergency department: effect on resource utilization. Archives of pediatrics \& adolescent medicine 156: 1108-1113.

12. Lion KC, Thompson DA, Cowden JD, Michel E, Rafton SA, et al. (2012) Impact of language proficiency testing on provider use of Spanish for clinical care. Pediatrics 130: e80-87. [Crossref]

13. Butt T, Khan HU, Ahmed I, Eldali A (2016) Emergency department attendance patterns during Ramadan. Annals of Saudi Medicine 36: 258.

Copyright: (C2019 Qureshi MN. This is an open-access article distributed under the terms of the Creative Commons Attribution License, which permits unrestricted use, distribution, and reproduction in any medium, provided the original author and source are credited. 